Short title: ELECTRODE PREPARATION OF CARBON SUPERCAPACITOS

\title{
INFLUENCE OF ELECTRODE PREPARATION ON THE ELECTROCHEMICAL BEHAVIOUR OF CARBON-BASED SUPERCAPACITORS
}

\author{
V. Ruiz, C. Blanco, M. Granda, R. Menéndez and R. Santamaría \\ Instituto Nacional del Carbón, CSIC \\ Apdo. 73, 33080-Oviedo (Spain)
}

Corresponding: Dr Clara Blanco

Instituto Nacional del Carbón, CSIC

Apdo. 73, 33080-Oviedo (Spain)

Tel. + 34985119090

Fax. + 34985297662

Email: clara@incar.csic.es 


\title{
INFLUENCE OF ELECTRODE PREPARATION ON THE ELECTROCHEMICAL BEHAVIOUR OF CARBON-BASED SUPERCAPACITORS
}

\author{
V. Ruiz, C. Blanco, M. Granda, R. Menéndez and R. Santamaría \\ Instituto Nacional del Carbón, CSIC \\ Apdo. 73, 33080-Oviedo (Spain)
}

Key words.- Activated carbon, binder, porosity, supercapacitors, solvent, surface area.

\begin{abstract}
.-
This paper investigates the influence of the electrode preparation on the electrochemical behaviour of carbon-based supercapacitors. Studies were performed using the same activated carbon and polymer (PVDF) in the same proportions (10 wt. \% PVDF). Only the way in which these components were mixed was modified. The procedure for mixing the activated carbon and the polymer in order to form the electrode has a significant influence on the electrochemical behaviour of the electrode used in a supercapacitor, as this determines the surface area accessible to the electrolyte. The mixing procedure can be selected in order to ensure optimum performance of the electrode. The use of NMP in the mixing procedure, the most common method reported in the literature, blocks a significant part of the porosity of the activated carbon, causing a decrease in the capacitance of the electrodes. The addition of the polymer using one of the other methods studied reduces the accessible surface area to a lesser extent, although the use of ball milling causes a decrease in the size of the carbon particles, which in turn increases the resistance of the electrode.
\end{abstract}




\section{Introduction}

Supercapacitors are an important alternative or complement to other energy storage or generation devices such as secondary batteries and fuel cells [1]. Carbon materials have been shown to be the most attractive electrode materials for these supercapacitors, as a consequence of their relatively low cost, high surface area and availability [2]. The manufacture of electrodes for supercapacitors requires the addition of a polymer to bind the carbon particles together. This procedure should ensure that the electrode is a compact pellet and that there is good electrical contact between the carbon particles. However, the addition of the polymer blocks part of the porosity of the carbon and also causes an increase in resistivity. The amount of polymer therefore should be kept as low as possible, in order not to reduce surface area or conductivity in the electrode. At the same time, enough polymer should be added to ensure that carbon particles are compact and so that the electrode is manageable. The most commonly used polymers are polyvynilidene fluoride (PVDF) [2], polyvynilidene chloride (PVDC) [3] and Teflon [4], in proportions that usually vary from 5 to $10 \mathrm{wt}$ \%.

In addition to the type of polymer and the amount used to make the electrode, the procedure used to mix it with the carbon may also significantly modify the final characteristics of the electrode, making it necessary to optimise the mixing stage. A number of methods for mixing the carbon and the polymer have been described in the literature. The most common make use of a solvent such as N-methyl-2-pyrrolidone (NMP) [5], tetrahydrofurane (THF) [3], acetonitrile [6], ethanol [7] or $\gamma$-butyrolactone [6]. A dry mixing of the carbon and the polymer, that does not involve the use of a solvent, has also been described in the literature [8, 9]. 
The wide range of possibilities for preparing the electrodes makes it difficult to compare the electrochemical data published by different research groups. Furthermore, the procedure for making the electrode and the characterisation of the resultant electrodes is missing in most of the cases. This paper aims to demonstrate how the different methods used to mix the polymer and the carbon may modify the characteristics of the resultant electrodes and, in turn, the behaviour of the electrodes in the supercapacitor. Studies were performed using the same activated carbon and polymer (PVDF) in the same proportions (10 wt. \% PVDF). Only the way in which the components were mixed was modified.

\section{Experimental}

An activated carbon (AC) obtained by chemical activation of naphthalene derived mesophase pitch AR24 was used as electrode active material. Activation was performed using a 3:1 $\mathrm{KOH}$ to carbon ratio, at $700^{\circ} \mathrm{C}$ for one hour, under a nitrogen flow of $62 \mathrm{~mL}$ $\min ^{-1}$. The resultant material was neutralized with $1 \mathrm{M} \mathrm{HCl}$ and then washed with distilled water until $\mathrm{pH}=7$. Finally, the carbon was dried at $110^{\circ} \mathrm{C}$ in a vacuum oven for $24 \mathrm{~h}$.

Carbon/PVDF electrodes were prepared using four different methods. Method 1 consisted of the manual mixing of the components in an agate mortar, followed by the moulding of the mixture to conform the electrodes. The electrodes obtained by this method were labelled M1. Method 2 involved the use of acetone to obtain a suspension of the activated carbon and the polymer, which were then mixed in an agate mortar and pressed into pellets using the same conditions as described before for method 1 . The electrodes obtained were labelled M2. Method 3 consisted in mechanically mixing the components in an agate ball mill Retsch, employing a frequency of $60 \mathrm{rpm}$ for 10 minutes. Two agate balls $12 \mathrm{~mm}$ in 
diameter were used for milling. The electrodes (M3) were obtained using the same moulding conditions as before. Method 4 is similar to method 2 but in this case NMP was used as solvent. The mixture tended to form agglomerates, as this solvent does not evaporate as easily as acetone. The mixture was therefore dried in the oven at $70^{\circ} \mathrm{C}$ for 30 minutes. The electrodes obtained after moulding were labelled M4.

The textural characteristics of the activated carbon (AC) and the electrodes prepared by the different methods were studied from the nitrogen adsorption isotherms at $77 \mathrm{~K}$. The measurements were performed in a volumetric system Micromeritics ASAP 2020. About $40 \mathrm{mg}$ of sample was used for each measurement. The electrodes were degasified prior to the analyses at lower temperatures $\left(100^{\circ} \mathrm{C}\right)$ than for the activated carbon $\left(300^{\circ} \mathrm{C}\right)$, to avoid the decomposition of the polymer. The specific surface area was determined from the $\mathrm{N}_{2}$ adsorption isotherms using the BET equation. The total micropore volume, the microporous surface area (Smic) and the average micropore diameter (Lo) were calculated by applying the Dubinin-Radushkevich (DR) equation to the $\mathrm{N}_{2}$ isotherm. The total pore volume was obtained from the amount of $\mathrm{N}_{2}$ adsorbed at $\mathrm{P} / \mathrm{P}_{0}=0.99$. Normalised $\mathrm{N}_{2}$ isotherms were obtained by dividing the nitrogen adsorbed at each pressure by the adsorption of nitrogen at $\mathrm{P} / \mathrm{P}_{0}=0.99$. Mesopore volume was calculated by subtracting the total micropore volume from the total pore volume. As the polymer does not contribute to adsorption, the volume of nitrogen adsorbed by each electrode was indicated by the mass unit of activated carbon in the electrode. 
The characteristic features of the electrodes were studied by SEM, using a Zeiss DSM-942 microscope. A backscattered electron detector was used to study the distribution of the polymer and activated carbon in each electrode.

The particle size of the samples after the mixing stage was determined in a Beckman Coulter LS 13320 analyzer, by means of an aqueous dispersion of the sample.

The electrochemical performance of the electrodes was studied by subjecting them to galvanostatic charge-discharge cycles in a two electrode Swagelok type cell using an aqueous solution of sulphuric acid (2 M) as electrolyte. The capacitor was constructed with two electrodes, separated by a disc of glassy paper. The galvanostatic charge-discharge characteristics were measured using an Autolab M30 coupled to an impedance spectroscopy module, in the range of $0-1 \mathrm{~V}$ at various current intensities (1-100 mA). The gravimetric specific capacitance was calculated from the linear region $(\sim 0.2-0.6 \mathrm{~V})$ of the discharge branch, taking into account the mass of activated carbon used for the lightest electrode. Impedance spectra were obtained in a frequency range of $1-100 \mathrm{kHz}$, with \pm 10 mV amplitude.

\section{Results and discussion}

The nitrogen adsorption isotherms of the activated carbon and the four electrodes are shown in Figure 1-a. The isotherm corresponding to the activated carbon is Type I, indicating that the sample is mainly microporous. The isotherms corresponding to the different electrodes are also Type I, which indicates that the type of porosity remains similar after the addition of the polymer. However, the ability of the electrodes to adsorb nitrogen decreases in all cases, adsorption being similar for electrodes M1, M2 and M3 but 
significantly lower for M4. It can be seen from Figure 1-b that the knee of the isotherms has changed in a similar way for the four types of electrodes. The addition of the polymer causes a widening of the knee, which is indicative of a wider pore size distribution.

The total volume of pores estimated from the isotherms (Table 1) decreases from $0.86 \mathrm{~cm}^{3}$ $\mathrm{g}^{-1}$ for the activated carbon to $0.71 \mathrm{~cm}^{3} \mathrm{~g}^{-1}$ for electrodes M1 to M3, it being only $0.59 \mathrm{~cm}^{3}$ $\mathrm{g}^{-1}$ for M4. The volume of micropores for the different samples (Table 1) follows a similar trend, decreasing from $0.79 \mathrm{~cm}^{3} \mathrm{~g}^{-1}$ (AC) to $0.61 \mathrm{~cm}^{3} \mathrm{~g}^{-1}$ (M1-M3) and then to $0.50 \mathrm{~cm}^{3} \mathrm{~g}^{-1}$ (M4). The percentage of microporosity also decreases, in a similar way for all the electrodes, which suggests that the polymer preferably blocks the access to the micropores, in agreement with the observations of Teng et al. [5]. This led to an increase in average pore diameter, which changed from $0.96 \mathrm{~nm}$ for AC to 1-1.1 nm for the electrodes.

These results corroborate that the method used to prepare the electrodes has a significant influence on the amount of surface accessible to the electrolyte. When NMP is employed (M4), the microporous surface area is reduced by about $46 \%$ with respect to the activated carbon, whereas for the other methods the reduction in area is around $24-29 \%$.

The distribution of the polymer and the activated carbon in each electrode was studied by SEM in order to explain their textural differences. Representative images of each sample are shown in Figure 2. The manual mixing of the components (method 1) yielded electrodes with a fairly homogeneous distribution of polymer and carbon (Figure 2-a). The polymer appears on the surface of the carbon particles, in the form of small circles. When acetone was used for the mixing (method 2), the polymer appears in the form of small spheres (Figure 2-b) i.e., the original form of the polymer. These spheres are not uniformly 
distributed in the electrode, but rather concentrated in cracks present in the carbon particles. This suggests that the acetone has dragged the polymer to these regions, where it has remained after the evaporation of the solvent. In the electrode obtained by ball-milling (M3), the particles of polymer cannot be distinguished from the carbon (Figure 2-c), which suggests that the polymer is finely and homogeneously distributed in the electrode as a consequence of the energetic mixing carried out in the mill. In fact, the particles of activated carbon are significantly smaller after the mixing stage. The average particle size decreased from $100 \mu \mathrm{m}\left(\mathrm{d}_{90}=200 \mu \mathrm{m} ; \mathrm{d}_{10}=31 \mu \mathrm{m}\right)$ for AC to $12 \mu \mathrm{m}\left(\mathrm{d}_{90}=14 \mu \mathrm{m} ; \mathrm{d}_{10}=2\right.$ $\mu \mathrm{m})$ after the ball milling. The particle size remained unchanged for the other methods studied. Finally, when NMP is used (M4), a homogeneous layer of polymer coating the carbon particles is observed (Figure 2-d), resulting from the dissolution of the polymer in the NMP, which did not occur with acetone. This coating of the carbon particles may be responsible for the significant reduction in the specific surface area of this electrode compared to the others.

The results obtained from the galvanostatic charge-discharge tests carried out with each electrode are shown in Figure 3. Electrodes M1 and M2 showed the highest capacitance values, which is not surprising considering their similar textural characteristics. The M4 electrodes showed the lowest capacitance values, because of their lower surface area. In fact, the decrease in capacitance is proportional to the decrease in the microporous surface area of the electrodes (around $27 \%$ ). The results obtained for the M3 electrodes are slightly lower than expected considering that the textural characteristics of M3 are very similar to those of M1 and M2. 
The results obtained by impedance spectroscopy are shown in Figure 4. As might be expected, Rs, which is related to the resistance of the electrolyte and the contacts between the current collectors and the electrodes, is the same for all the samples. Meanwhile, the values of Rf, which are related to the intrinsic resistance of the electrode, show some differences. Electrode M4 has a significantly higher resistance than the others, which is the result of the poorer contact between carbon particles because they are coated by the polymer. The resistances of M1 and M2 are very similar and slightly lower than that of M3. This result may be related to the smaller particle size of M3 which is a result of the mixing in the ball mill, and might explain the slightly lower capacitance values observed for this sample in relation to M1 and M2.

\section{Conclusions}

The procedure for mixing the activated carbon and the polymer in order to form the electrode has a significant influence on the electrochemical behaviour of the electrode in a supercapacitor, as it determines the surface area accessible to the electrolyte. The mixing procedure can be selected in order to ensure optimum electrode performance. The use of NMP in the mixing procedure, the most common method reported in the literature, blocks a significant part of the porosity of the activated carbon, causing a decrease in electrode capacitance. The addition of polymer using one of the other three methods studied reduces the accessible surface area in a similar way, although the use of ball milling causes a decrease in carbon particle size and this in turn increases the resistance of the electrode. 


\section{Acknowledgements}

This work was been performed with financial support from MEC (project MAT200403480-C02) and FICYT (project IB05-086-C1). V. Ruiz acknowledges a predoctoral research grant from FICYT. 


\section{References}

[1] R Kötz and M. Carlen, Electrochimi Acta 45 (2000) 2483-2498.

[2] E. Frackowiak and F. Béguin, Carbon 39 (2001) 937-950.

[3] K. Hyeok, W. Sek Kim and Y. Soon Park, Advanced Materials 13 (2001) 497-500.

[4] S. Shiraishi, H. Kuriharea and A. Oya, Carbon Sicence 1 (2001) 133-137.

[5] H. Teng, Y-J Chang and C-T Hsieh, Carbon 39 (2001) 1981-1987.

[6] A. Celzard, F. Collas, J.F. Marêché, G. Furdn and I. Rey, J Power Sources 108 (2002) 153-162.

[7] N-L. Wu, S-Y. Wang and C-Y. Han, J Power Sources 113 (2003) 173-178.

[8] Y-J. Kim, Y. Horie, Y. Matsuzawa, S. Ozaki, M. Endo and M.S. Dresselhaus, Carbon 42 (2004) 2423-2432.

[9] A. Alonso, V. Ruiz, C. Blanco, R. Santamaría, M. Granda, R. Menendez and S.G.E. de Jager, Carbon 44 (2006) 441-446. 
Table 1. Textural characteristics of the activated carbon and the different electrodes.

\begin{tabular}{cccccccc}
\hline & $\begin{array}{c}\mathrm{V}_{\mathrm{t}} / \\
\mathrm{cm}^{3} \mathrm{~g}^{-1}\end{array}$ & $\begin{array}{c}\mathrm{V}_{\mathrm{N} 2} / \\
\mathrm{cm}^{3} \mathrm{~g}^{-1}\end{array}$ & $\begin{array}{c}\mathrm{L}_{0} / \\
\mathrm{nm}\end{array}$ & $\begin{array}{c}\mathrm{S}_{\mathrm{mic}} / \\
\mathrm{m}^{2} \mathrm{~g}^{-1}\end{array}$ & $\begin{array}{c}\mathrm{S}_{\mathrm{BET}} / \\
\mathrm{m}^{2} \mathrm{~g}^{-1}\end{array}$ & $\begin{array}{c}\mathrm{V}_{\text {meso }} / \\
\mathrm{cm}^{3} \mathrm{~g}^{-1}\end{array}$ & $\begin{array}{c}\text { Microprosity/ } \\
\%\end{array}$ \\
\hline AC & 0.86 & 0.79 & 0.96 & 1642 & 1963 & 0.07 & 92 \\
M1 & 0,71 & 0,61 & 1,03 & 1174 & 1593 & 0,10 & 86 \\
M3 & 0,71 & 0,61 & 1,01 & 1210 & 1623 & 0,10 & 86 \\
M4 & 0,71 & 0,61 & 0,98 & 1241 & 1643 & 0,10 & 86 \\
\hline
\end{tabular}

$\mathrm{V}_{\mathrm{t}}$, total volume of pores

$\mathrm{V}_{\mathrm{N} 2}$, volume of micropores

$\mathrm{L}_{0}$, average pore diameter

$\mathrm{S}_{\text {mic }}$, microporous surface area

$\mathrm{S}_{\mathrm{BET}}$, apparent surface area BET

$\mathrm{V}_{\text {meso, }}$, volume of mesopores 


\section{Figure Captions}

Figure 1.- a) $\mathrm{N}_{2}$ adsorption isotherms and b) normalised isotherms of activated carbon AC and the different electrodes.

Figure 2.- SEM images of the different electrodes: a) M1; b) M2; c) M3 and d) M4.

Figure 3.- Variation of specific discharge capacitance with current density for the different electrodes.

Figure 4.- Impedance spectra obtained for the different electrodes. 


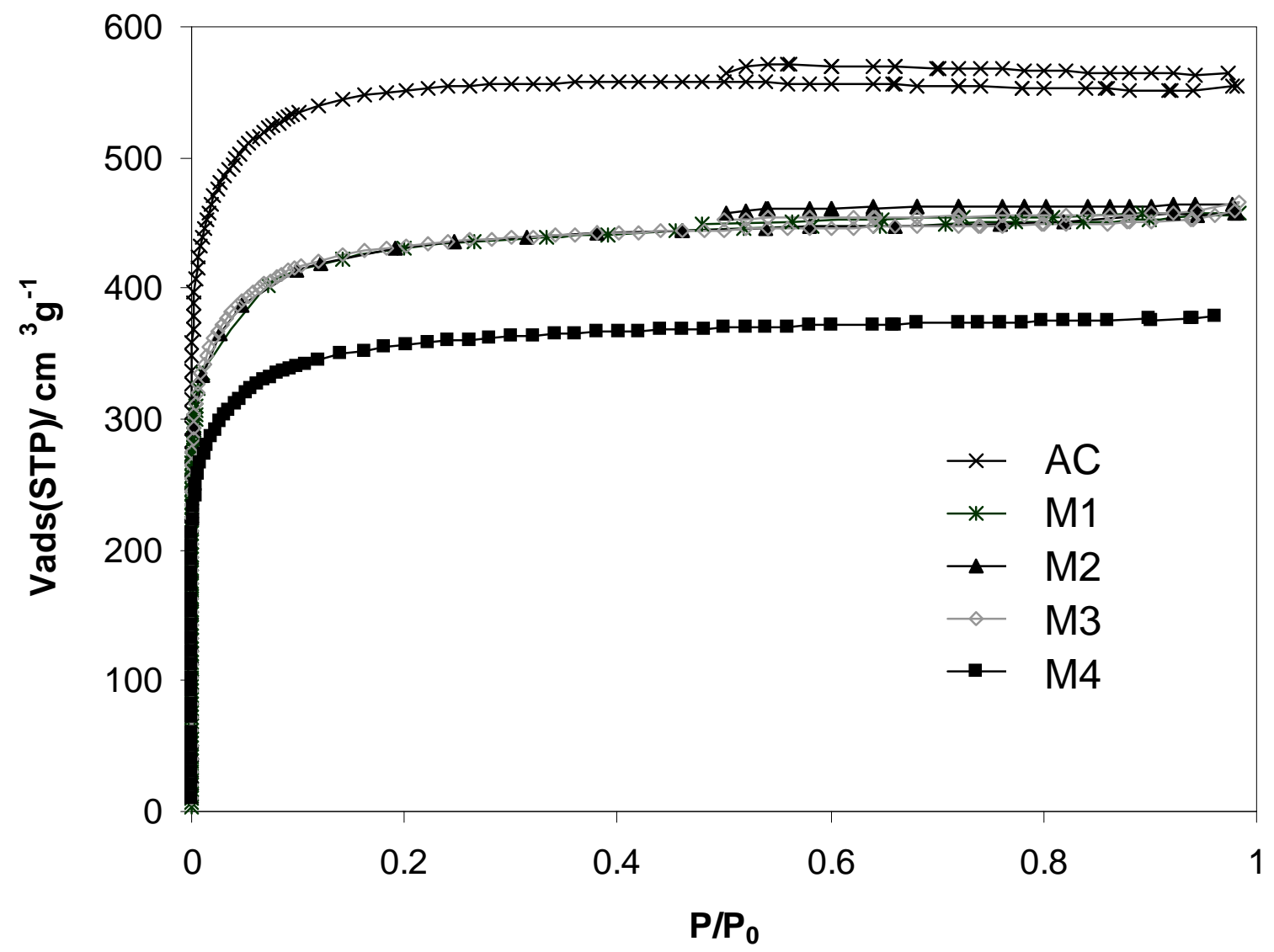

Figure $1 \mathrm{a}$ 


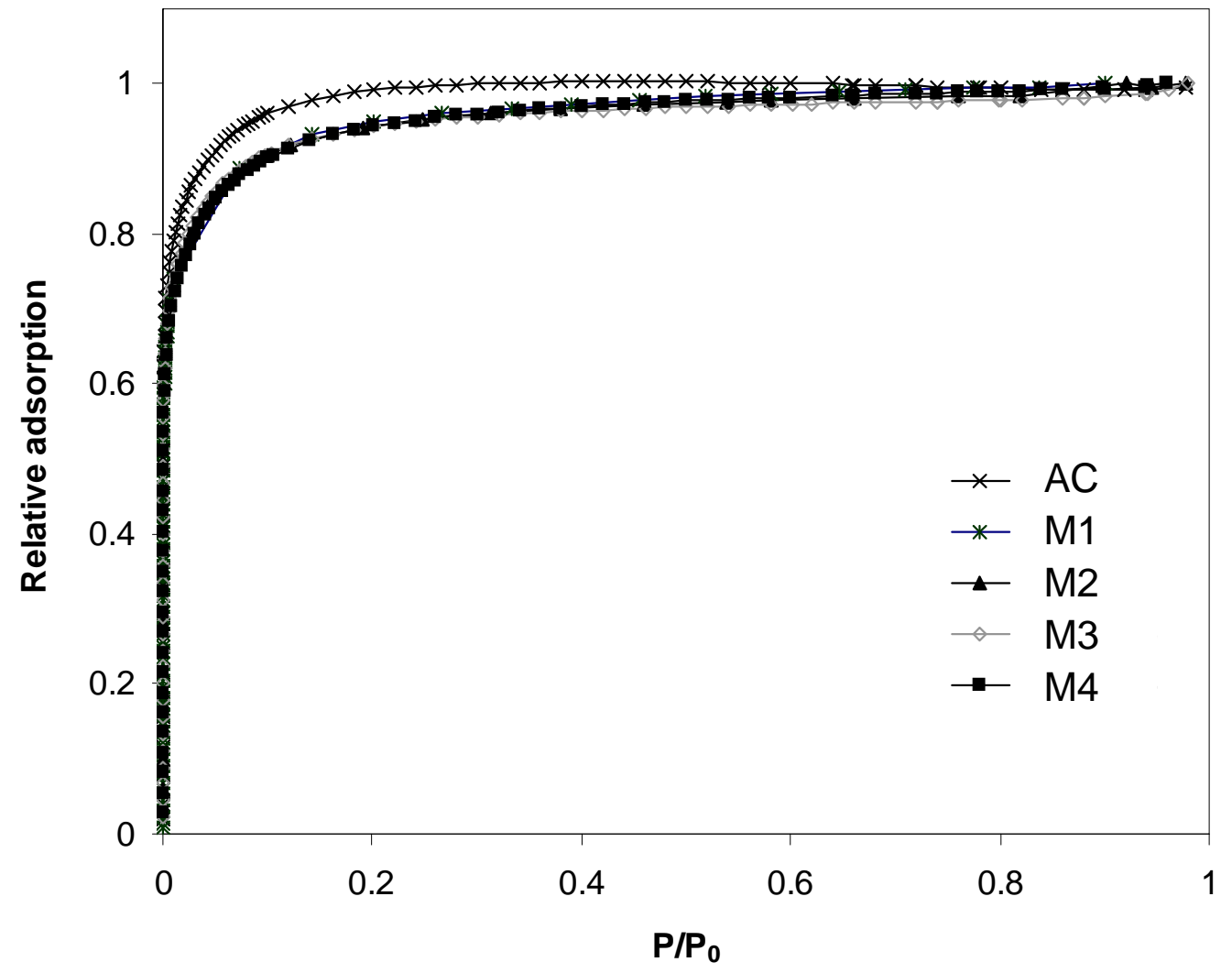

Figure $1 \mathrm{~b}$ 

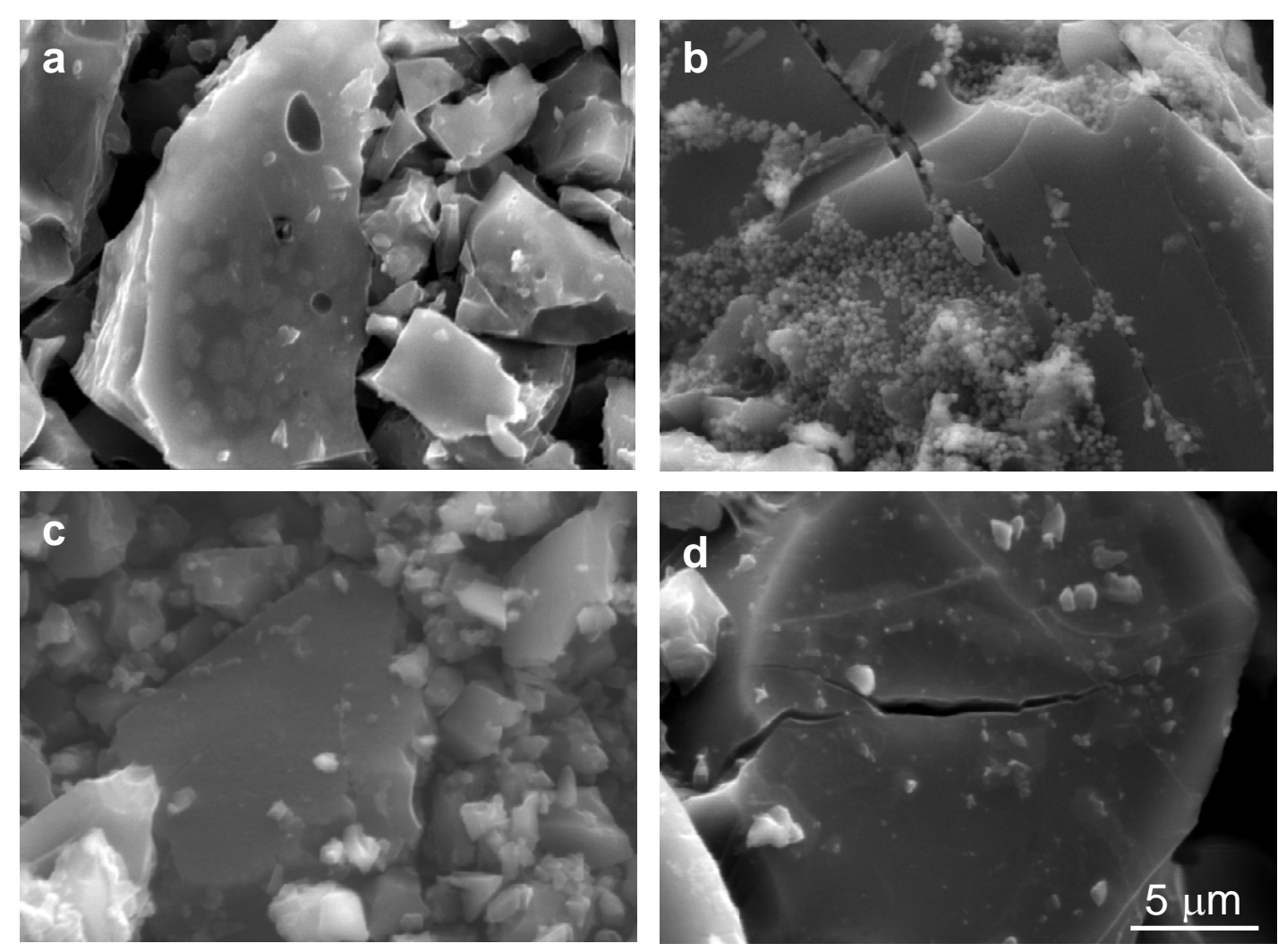

Figure 2 


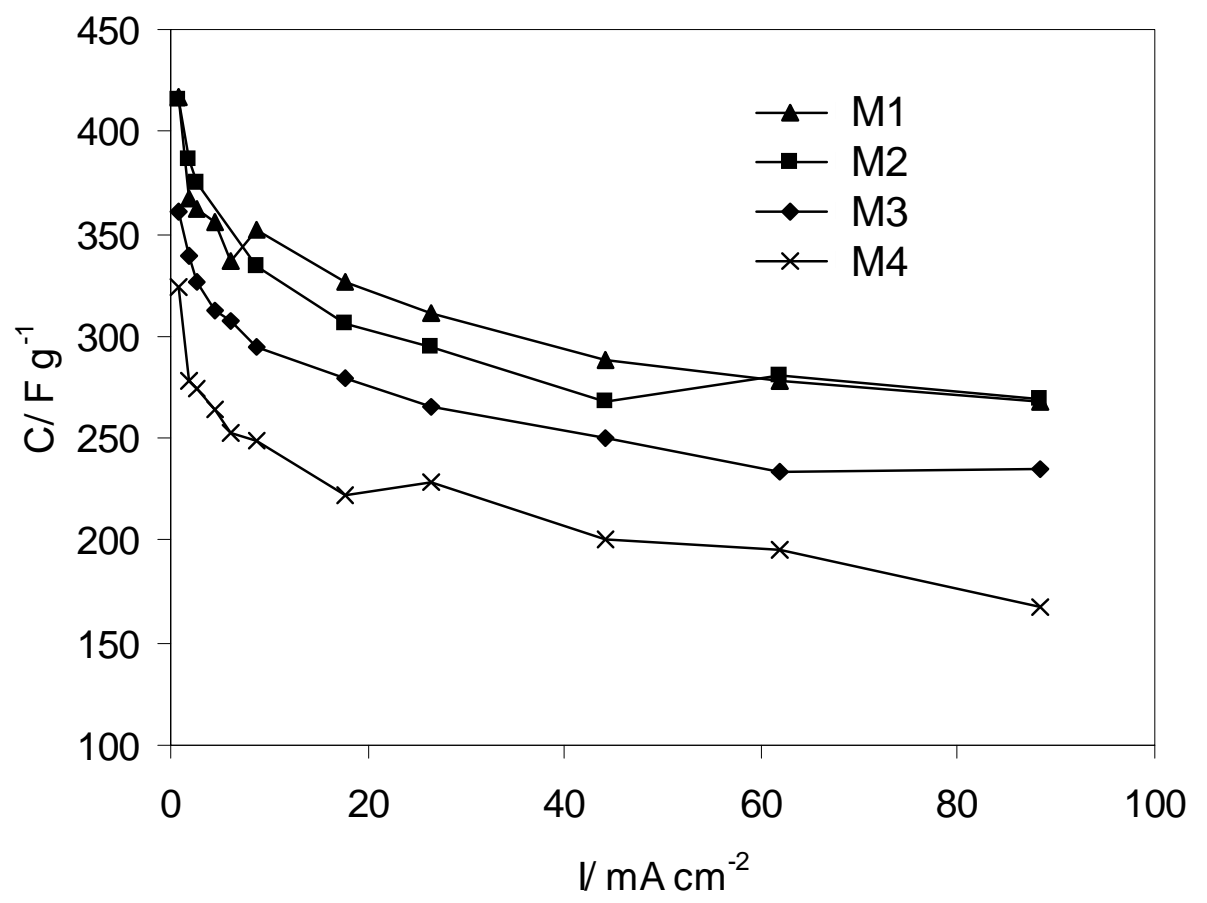

Figure 3 


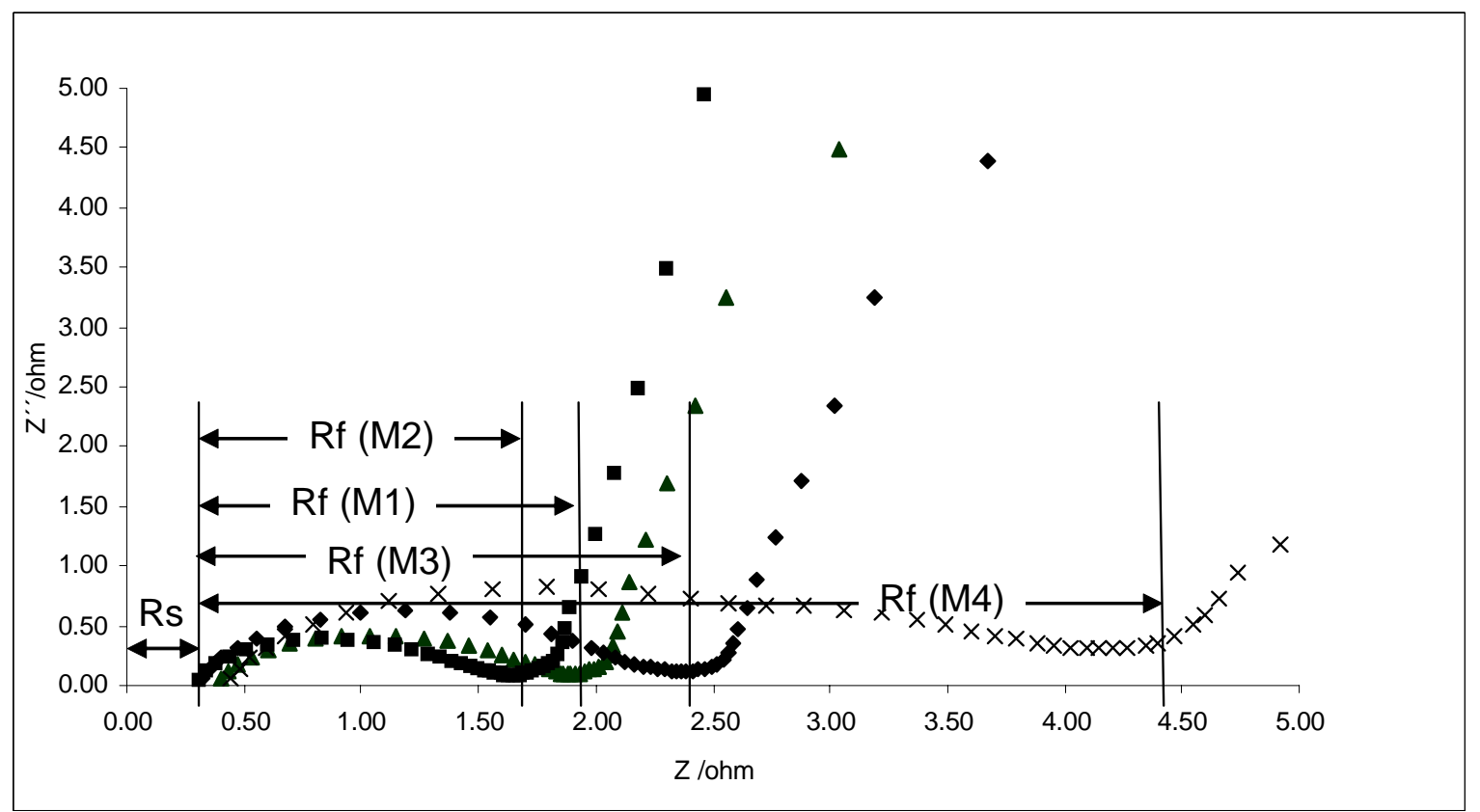

Figure 4 